\title{
Exciton spectroscopy of red polydiacetylene chains in single crystals
}

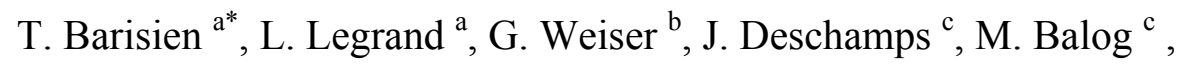 \\ B. Boury ${ }^{\text {c }}$, S.G. Dutremez ${ }^{\text {c }}$, M. Schott ${ }^{\text {a }}$
}

${ }^{\text {a }}$ Institut des NanoSciences de Paris, UMR7588 CNRS / Universités Pierre et Marie Curie (Paris 6) et Denis Diderot (Paris 7), Campus Boucicaut, 140 rue de Lourmel, 75015 Paris, France

\footnotetext{
${ }^{\mathrm{b}}$ Faculty of Physics and Centre of Material Science, Philipps-Universität, 35037 Marburg, Germany

${ }^{\mathrm{c}}$ Institut Charles Gerhardt Montpellier, UMR 5253 CNRS-UM2-ENSCM-UM1, Université Montpellier II,

Bât. 17, CC 1700, Place Eugène Bataillon, 34095 Montpellier Cedex 5 France
}

* Corresponding author. Fax 33143542878

E-mail address: Thierry.Barisien@insp.jussieu.fr 


\begin{abstract}
The absorption and emission properties of excitons are investigated in a pure "red" phase of luminescent polydiacetylene wires. The values of the binding energy $\left(E_{b}=0.556 \mathrm{eV}\right)$ and Bohr radius $(r \sim 14 \AA)$ make the "red" exciton similar to the one of the extensively studied "blue" phase, so the conformational modifications which lead to a reordering of excited states and luminescent emission do not strongly affect the exciton structure. The opening of a thermally activated non radiative channel leads to an efficient quenching of the intense luminescence observed at low temperature.
\end{abstract}

\title{
Introduction
}

Polydiacetylene (PDA) chains exist in the solid state as either of two electronic structures commonly called the blue and red phases. Single isolated chains of red phase poly-3BCMU have remarkable electronic properties. A chain behaves like a quasi-perfect one-dimensional system [1] with the spatial coherence of the red exciton extending over macroscopic distances [2]. However, in these crystals, red chains coexist with a majority population of blue chains. The red chains can be discriminated from the weakly emitting blue ones only because of their strong luminescence. The coexistence of two populations of chains in the same crystal is a limiting factor since the absorption properties of the red polydiacetylenic structure cannot be selectively probed. For instance photo-induced absorption experiments which have allowed the investigation of the relaxation dynamics of the blue phase exciton are impossible $[3,4,5]$.

The differences in electronic properties of each phase are necessarily associated to modifications of the ground state structure. As the current data from X-ray diffraction experiments do not discriminate between red and blue geometries, the structural differences in 
the ground state must be quite small. The only results showing clear variations are from solid state ${ }^{13} \mathrm{C}$ NMR spectroscopy $[6,7]$. Determination of the red chain conformation is thus a still unresolved issue which is related to the general question of the relationship between electronic configuration and structural parameters in conjugated polymers.

Given this situation, we have searched for a purely red system of PDA chains with the further requirement of being able to achieve sufficiently low polymer contents in a good crystallographic environment. The spectroscopic results presented in this letter indicate that the polydiacetylene chain obtained from the polymerization of 1,6-bis(diphenylamino)-2,4hexadiyne (THD) leads to the formation of a purely red phase, promising for comparative studies between the two conformational isomers of PDA. Up to now the structural and electronic properties of poly-THD have been addressed in a limited number of papers. The room temperature crystal structure has been determined [8]. Room temperature reflectance and Raman scattering spectra have been measured by Morrow [9]. The Kramers-Kronig transformation of the reflectance yields a zero phonon absorption band peaked at $568 \mathrm{~nm}$ and characteristic of a red PDA phase. The fluorescence spectra of thin films made of microcrystallites dispersed in a PMMA matrix have also been measured [10]. So far, all of the studies have dealt with fully or almost fully polymerized crystals.

In this work, the exciton spectroscopy of highly ordered poly-THD chains dispersed in their monomer crystal is investigated at low temperature. As previous reports indicated a strong thermal reactivity of crystalline THD, particular attention has to be paid to avoid thermal reaction. In our work, the polymer content in weight, $\mathrm{x}_{\mathrm{p}}$, was kept around a few percent except for reflectance and electroreflectance experiments where $\mathrm{x}_{\mathrm{p}}$ was $\sim 65 \%$, as inferred by the absolute value of the reflectance and the sum rule of the oscillator strength (see fig. 1). 


\section{Experimental}

1,6-Bis(diphenylamino)-2,4-hexadiyne (THD) was prepared in two steps starting from commercial diphenylamine. In the first step, lithium diphenylamide was allowed to react with stoichiometric amounts of propargyl bromide, in THF, at $-78^{\circ} \mathrm{C} . \mathrm{N}, \mathrm{N}-$ Diphenylpropargylamine was obtained as a white crystalline solid after purification by vacuum distillation and column chromatography (yield $=50 \%$ ). THD was obtained in $65 \%$ yield by oxidative dimerization of $N, N$-diphenylpropargylamine $[11,12,13] .{ }^{1} \mathrm{H} N M R,{ }^{13} \mathrm{C}$ NMR and IR data fully agree with the expected molecular structure and previous literature reports [14]. Monomer crystals were grown from a 4:1 methylene chloride - diethyl ether mixture $(\mathrm{c} \sim 15 \mathrm{~g} / \mathrm{l})$, at $0^{\circ} \mathrm{C}$, in the dark. The crystals are needle-shaped with a width of a fraction of millimetre, the chains being oriented along the main dimension.

Polarized reflectance spectra in the energy range between 1.5 and $5.8 \mathrm{eV}$ were obtained from flat areas of about $0.5 \mathrm{~mm} \times 1 \mathrm{~mm}$ in size using a windowless vacuum spectrometer and calcite polarizers. For electroreflectance measurements, $\mathrm{Cr} / \mathrm{Mg}$ contacts spaced by a $0.5 \mathrm{~mm}$ distance were evaporated to apply along the polymer backbone an external sinusoidal voltage at $1 \mathrm{kHz}$ frequency. The field modulated light was collected by lock-in at twice the field frequency and evaluated as described in previous studies [15]. Absorption spectra were derived by Kramers-Kronig transformation for comparison with absorption for light polarized perpendicular to the chain. The latter spectra were measured in the double beam mode of a Cary 5000 double monochromator using freshly prepared samples with low polymer content and selecting $0.1 \mathrm{~mm}$ wide homogeneous areas. The spectral resolution was $4 \AA$ for reflectance and transmission measurements and $5 \AA$ for electroreflectance spectra.

Raman spectra were measured using a Jobin Yvon U1000 double monochromator and the excitation was the $647.1 \mathrm{~nm}$ line of a mixed $\mathrm{Ar}+/ \mathrm{Kr}+$ laser. For both Raman and absorption 
measurements the samples were mounted on the cold finger of a $\mathrm{He}$ gas exchange cryostat that allows studies down to $10 \mathrm{~K}$.

The emission was studied in a confocal-like configuration. Detailed description of the set-up can be found elsewhere [16].

\section{Results and discussion}

\section{Reflectance and electroreflectance}

The reflectivity of the samples in the visible region is very anisotropic. For light polarized perpendicular to the polymer chain, the reflectivity shows no structure and remains below $5 \%$ up to $3.8 \mathrm{eV}$ where the absorption by the phenyl side-groups sets in. For parallel polarization of light, the room temperature spectrum in fig. 1 shows a broad exciton peak of about $30 \%$ reflectance followed by a vibrational band as reported earlier [9]. Low temperature reflectance spectra show some narrowing of the main peak, and a manifold of vibronic exciton peaks appears as is typically observed in high-quality polydiacetylene single crystals $[15,17]$.

Much more structure is resolved in low temperature electroreflectance (ER) spectra (fig.1). Two spectra scaled quadratically to the fields of 15 and $30 \mathrm{kV} / \mathrm{cm}$ (full and dashed lines respectively) are almost identical. This proves that all features below $\sim 2.6 \mathrm{eV}$ increase quadratically with field strength and are reproduced in their spectral position and shape by the first derivative of the reflectivity, displayed on top by the dotted line. The electroreflectance spectrum thus reveals a rigid redshift of the exciton and its vibronic satellites by a quadratic Stark effect. The shift in a field of $15 \mathrm{kV} / \mathrm{cm}$ is about $8 \mu \mathrm{eV}$, similar to that observed in other polydiacetylene crystals $[15,18]$. The derivative spectrum does not reproduce the large signal above $2.7 \mathrm{eV}$ which increases sublinearly at high fields. This feature arises from the FranzKeldysh (FK) effect of the exciton continuum [15, 19]. The large signal at small fields indicates the excellent quality of the investigated area since it can be observed only if the 
scattering rate is small enough to allow a free electron hole pair to gain significant energy in an external field $[15,20]$.

Kramers-Kronig transformation yields, with an accuracy better than $5 \mathrm{meV}$, the exciton transition energy, $2.171 \mathrm{eV}$ and the free carrier gap, $2.727 \mathrm{eV}$, indicating an exciton binding energy $\mathrm{E}_{\mathrm{b}} \sim 556 \mathrm{meV}$ (see table 1). This is about 10\% larger than that obtained in bulk blue PDA crystals with exciton below $2 \mathrm{eV}$, but similar to that of red bulk crystals like TCDU [21]. The derivative lineshape of the ER spectrum is compatible with coupling of the exciton by the electric field $\mathrm{F}$ to its continuum of ionized states with the mean square displacement $\mathrm{r}$ of electron and hole representing the transition dipole [15]:

$$
\Delta \mathrm{E}=\frac{|\mathrm{e} \cdot \overrightarrow{\mathrm{r}}|^{2}}{\mathrm{E}_{\mathrm{exc}}-\mathrm{E}_{\mathrm{g}}}
$$

Inserting the experimental values yields an exciton radius of $14 \AA$, slightly larger than that derived for the blue chains of DCHD [15]. The exciton ground state thus extends over 5 diacetylene repeat units and must be delocalized on the chain. "Blue" and "red" excitons are intrinsically similar states, with internal structure quasi-independent of the electronic structure of the chain. Note that presently, due to interfering contributions of band edges corresponding to vibronic exciton replicae to the FK signal, a reliable determination of the coherence length of continuum states could not be deduced. Its amplitude is nevertheless of the same order than in bulk DCHD crystals [15], indicating highly ordered THD chains along which coherent motion occurs.

\section{Absorption and luminescence}

Thermal reactivity of the THD crystal is so high that the polymer content at the end of the sample growth precludes absorption in parallel polarization to be measured. We thus compare in figure 2 the experimental absorption for a polarization of the field perpendicular to the 
chain axis, $\alpha_{\perp}$, to the calculated absorption obtained by Kramers Kronig transformation of the reflectance shown in fig. $1 . \alpha_{\perp}$ reaches only $100 \mathrm{~cm}^{-1}$ for a value of $x_{p}$ estimated to be $2-3 \%$, which confirms the strong anisotropy by more than 2 orders of magnitude of $\pi-\pi *$ absorptions. The larger linewidth of the exciton peak at high energy in parallel polarization is due to the rapid decrease of the refractive index $\mathrm{n}$ above the exciton resonance and is absent in spectra of the dielectric constant, $\operatorname{Im}(\varepsilon)=n c \alpha / \omega$. The arrow indicates the energy gap which, due to strong Coulomb coupling of electron and hole, does not have sufficient strength to be resolved in absorption under the excitonic progression.

The luminescence spectrum is well resolved and is nearly a mirror image of the absorption. The inhomogeneous broadening of the zero phonon line is $\sim 40 \mathrm{meV}$ at $10 \mathrm{~K}$, comparable to the absorption linewidth. It is affected by re-absorption and cannot be considered as an origin for the emission. This origin is recovered by adding the energy of ground states normal modes measured by Raman spectroscopy to the corresponding vibronic lines in the emission. Using the $\mathrm{C}=\mathrm{C}$ and $\mathrm{C} \equiv \mathrm{C}$ stretching modes as values (fig. 3) places the origin at $2.153 \mathrm{eV}$, that is $\sim 18 \mathrm{meV}$ below the exciton energy deduced from electroreflectance. This Stokes shift cannot be associated to diffusion of excitons to low energy sites since ER data imply a highly regular chain similar to poly-3BCMU red chains. The presence of such sites would indeed suggest a disordered state of the chains, which is inconsistent with the observation of the FK effect in the field modulated signals. It thus corresponds to a real shift between the emission and absorption origins. No such Stokes shift is observed in the red phase of highly diluted poly3BCMU chains [22] and its origin in poly-THD is under study.

The emission of crystals with low polymer content is surprisingly strong. Given that the geometry of the collecting optics does not differ from one experiment to the other, it is possible to compare the emission intensities of poly-THD and poly-3BCMU isolated chains, the yield of which is known [23]. A rough estimate of the quantum yield, $\eta_{\mathrm{F}}$, leads to 
$\eta_{\mathrm{F}}(\mathrm{THD})>0.6$ at $10 \mathrm{~K}$. An extremely fast drop in the luminescence intensity with increasing temperature is also observed; this phenomenon follows an Arrhenius-type law with a thermal activation energy $E_{\text {act }} \sim 4.3 \mathrm{meV}$ (fig. 4). This corresponds to the opening of a non radiative channel. The origin of this process is not known yet but it seems to be a generic property of the red structure. Isolated poly-3BCMU chains indeed show a similar behaviour above $50 \mathrm{~K}$, with a much higher activation energy, $\mathrm{E}_{\mathrm{act}} \sim 38 \mathrm{meV}[24]$.

\section{Discussion and conclusions}

The present results can be compared to those obtained on red and blue poly-3BCMU chains. At the lowest temperature, poly-THD is more fluorescent than these red chains, and much more than any other PDA red phase. However, the poly-THD exciton energy is lower by $\sim 150 \mathrm{meV}$ than that of red poly-3BCMU, and its Raman frequencies are also intermediate between those of red and blue poly-3BCMU.

The blue chain is planar and the red one cannot be so [25]. A model in which successive repeats units are alternatively tilted out of the average plane by $+\theta$ and $-\theta$ angles is reasonable, and furthermore agrees well with NMR results [6, 7]. The unit cell of the red chain thus contains two non-translationaly equivalent monomer units. In poly-3BCMU, sidegroups are linked by hydrogen bonds which certainly influence the value of $\theta$. In poly-THD there are not such bonds and successive repeat units differ in the packing $(\mathrm{C}-\mathrm{H} \ldots \pi$ interactions) of phenyl groups. This may lead to a different $\theta$ value.

Simple considerations show that increasing $\theta$ decreases conjugation, hence increases the energy of the excited states. The value of $\theta$ in poly-THD would then be smaller than in poly3BCMU. Different excited states shift differently, and the occurrence of fluorescence may correspond to a much faster shift of the "dark" $\mathrm{A}_{\mathrm{g}}$ states which are present in the optical gap of blue chains [3], so that in red ones they lay above the optical gap. 
The activated decay of fluorescence yields may thus be associated with an activated transfer of the excitonic population to this $\mathrm{A}_{\mathrm{g}}$ state. However, the quenching mechanism can also imply a virtual state positioned slightly above the gap and corresponding to the dissociation threshold of a singlet exciton in two triplets, as observed in blue chains [26]. In the latter case, delayed luminescence should result associated to the long lifetime of the triplets. Time resolved luminescence experiments will give an insight into the nature of the process.

Poly-THD chains thus form quantum wires, the excitonic properties of which, on the whole, compare well with those of isolated poly-3BCMU red chains. The observation of an intense Franz-Keldysh effect in the electroreflectance spectra is the signature of a mixed monomerpolymer crystalline phase of "red" nature possessing a high degree of order. The latter appears as a promising model system of $1 \mathrm{D}$ crystals, in particular for the investigation, through timeresolved non-linear optical spectroscopy, of how the electronic structure is affected by the conformations in the frame of strongly interacting electrons.

\section{Acknowledgments}

We wish to thank Dr. J.-F. Mohrange for his assistance in Raman Scattering experiments. G. Weiser thanks the INSP for its hospitality. This work was funded in part by the Agence Nationale de la Recherche through contract ANR-06-NANO-013-02.

\section{References}

[1] F. Dubin, J. Berréhar, R. Grousson, T. Guillet, C. Lapersonne-Meyer, M. Schott, V. Voliotis Phys. Rev. B 66 (2002) 113202.

[2] F. Dubin, R. Melet, T. Barisien, R. Grousson, L. Legrand, M. Schott, V. Voliotis Nature Phys. 2 (2006) 32.

[3] B. Kraabel, M. Joffre, C. Lapersonne-Meyer, M. Schott Phys. Rev. B 58 (1998) 15777. 
[4] J.-Y. Bigot, T.-A. Pham, T. Barisien Chem. Phys. Lett. 259 (1996) 469.

[5] Y. Yuasa, M. Ikuta, T. Kobayashi Phys. Rev. B 72 (2005) 134302.

[6] A.L. Cholli, D.J. Sandman, W. Maas Macromolecules 32 (1999) 4444.

[7] H. Tanaka, M.A. Gomez, A.E. Tonelli, M. Thakur Macromolecules 22 (1989) 1208.

[8] V. Enkelmann, G. Schleier Acta. Crystallogr. B 36 (1980) 1954.

[9] M.E. Morrow, K.M. White, C.J. Eckhardt, D.J. Sandman Chem. Phys. Lett. 140 (1987) 263.

[10] N.V. Agrinskaya, E.G. Guk, I.A. Kudryavtsev, O.G. Lyublinskaya Phys. Solid State 37 (1995) 526.

[11] A. S. Hay J. Org. Chem. 27 (1962) 3320.

[12] L. Brandsma in Preparative Acetylenic Chemistry, Second Edition, Elsevier, Amsterdam, 1988.

[13] B. N. Ghose Synth. React. Inorg. Met. Org. Chem. 24 (1994) 29.

[14] A. Sharifi, M. Mirzaei, M.R. Naimi-Jamal J. Chem. Res. (S) (2002) 628.

[15] G. Weiser Phys. Rev. B 45 (1992) 14076.

[16] T. Guillet, J. Berréhar, R. Grousson, J. Kovensky, C. Lapersonne-Meyer, M. Schott, V. Voliotis Phys. Rev. Lett. 87 (2001) 087401.

[17] A. Horvath, G. Weiser, C. Lapersonne-Meyer, M. Schott, S. Spagnoli Phys. Rev. B 53 (1996) 13507.

[18] Y. Tokura, T. Koda, A. Itsubo, M. Miyabashi, K. Okuhara, A. Ueda, J. Chem. Phys. 99 (1986) 85 .

[19] G. Weiser and A. Horvath in Primary photoexcitations in conjugated polymers, N.S. Sariciftci (editor), World Scientific, Singapore 1997, chapter 12.

[20] A. Jaeger, G. Weiser Phys. Rev. B 58 (1998) 10674.

[21] Y. Tokura, Y. Oowaki, T. Koda Chem. Phys. 88 (1984) 437. 
[22] R. Lécuiller, J. Berréhar, C. Lapersonne-Meyer, M. Schott Phys. Rev. Lett. 80 (1998) 4068 .

[23] R. Lécuiller, J. Berréhar, C. Lapersonne-Meyer, M. Schott, J.D. Ganière Chem. Phys. Lett. 314 (1999) 255.

[24] R. Lécuiller, J. Berréhar, J.D. Ganière, C. Lapersonne-Meyer, P. Lavallard, M. Schott Phys. Rev. B 66 (2002) 125205.

[25] M. Schott J. Phys. Chem. B 110 (2006) 15864.

[26] B. Kraabel, D. Hulin, C. Aslangul, C. Lapersonne-Meyer, M. Schott Chem. Phys. 227 (1998) 83.

\section{Figure captions}

Figure 1: Reflectance and electroreflectance spectra with light polarized parallel to the polymer chain. The ER spectrum obtained for $30 \mathrm{kV} / \mathrm{cm}$ is reduced in size by a factor 4 . The dotted line shows the derivative of the reflectivity.

Figure 2: Absorption spectra with polarization parallel (Kramer Kronig transform) and perpendicular (experimental) to the polymer chain, derived from the reflectance and transmission spectra respectively. The luminescence at $10 \mathrm{~K}$ (dots) is for a $488.0 \mathrm{~nm}$ continuous wave excitation and a sub-microwatt pump fluence.

Figure 3: Typical low temperature Raman spectrum of a THD crystal having low polymer content $\left(\mathrm{x}_{\mathrm{p}} \leq 3 \%\right)$. The excitation power is $10 \mathrm{~mW}$ at $647.1 \mathrm{~nm}$. The frequency of the double bond and triple bond stretching modes are respectively $1481 \pm 2 \mathrm{~cm}^{-1}$ and $2107 \pm 2 \mathrm{~cm}^{-1}$. For a detailed discussion of the attribution of the other lines see [9]. 
Figure 4: Luminescence spectra at $11.3 \mathrm{~K}$ (solid line), $16.0 \mathrm{~K}$ (dots) and $44.0 \mathrm{~K}$ (dashes) excited at $488.0 \mathrm{~nm}$ for a polarization of excitation parallel to the chains. Inset: Arrhenius plot of the luminescence integrated over the 566 - $800 \mathrm{~nm}$ range (squares) and the associated linear fit. 


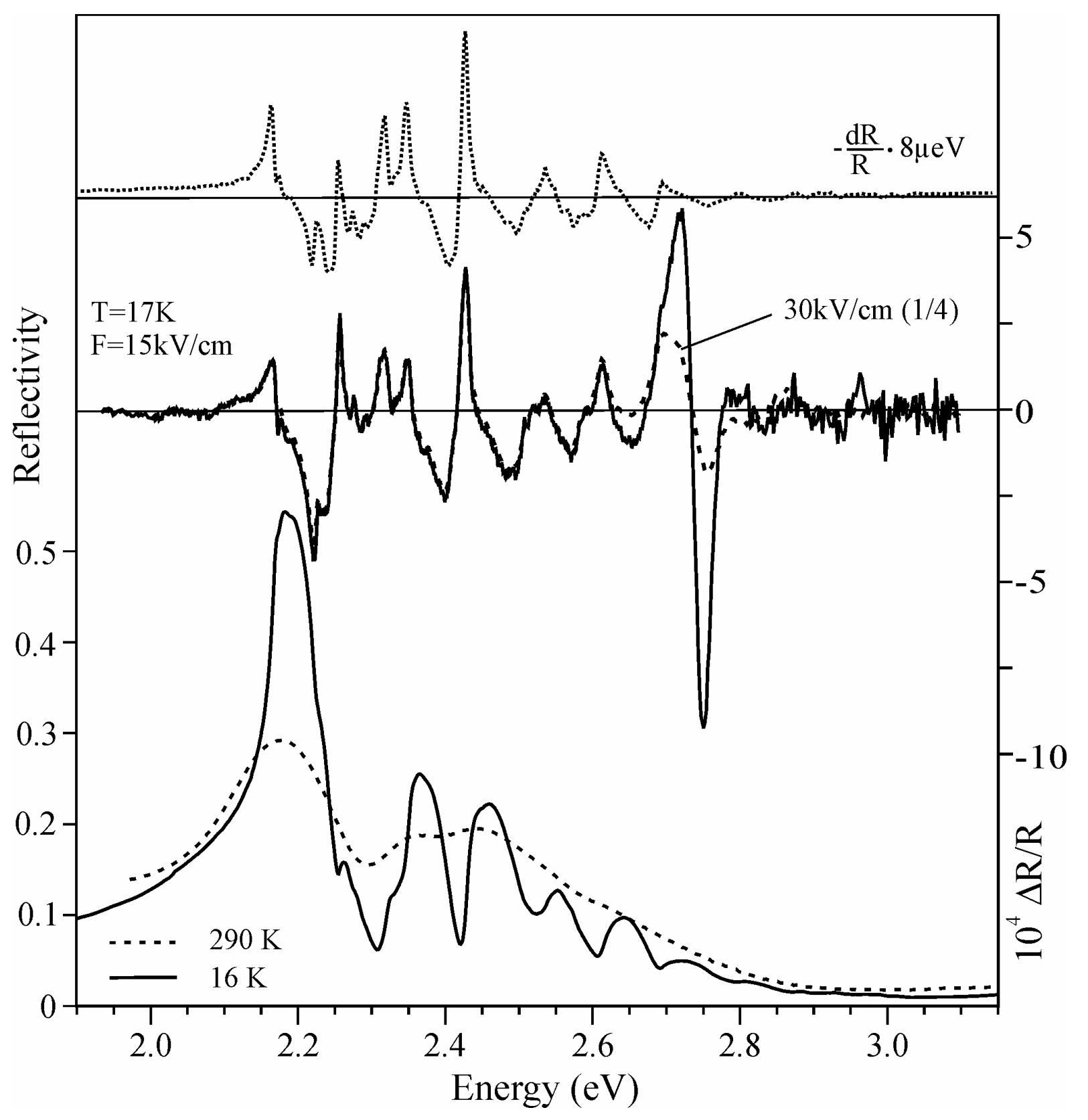

Figure 1 


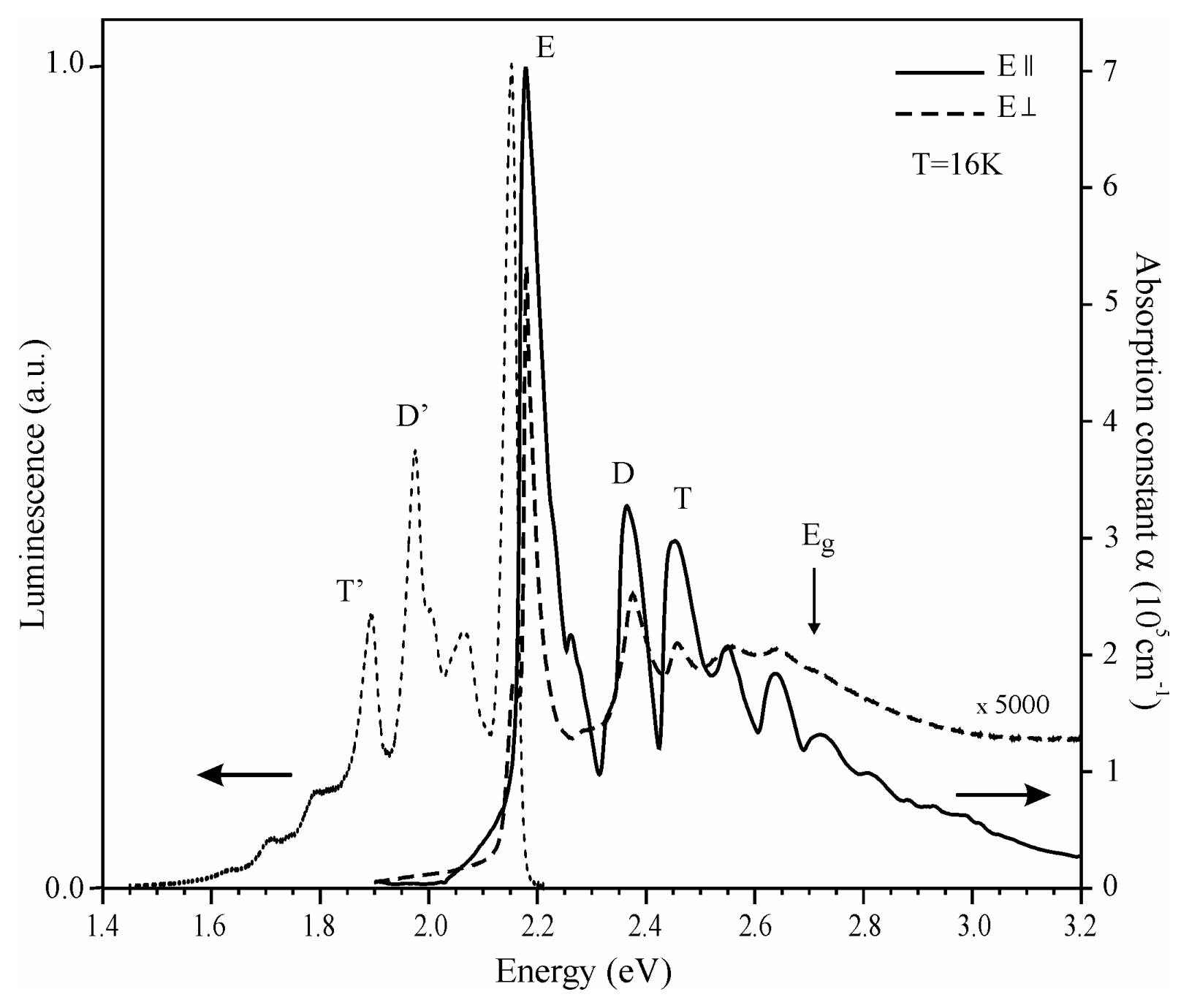

Figure 2 


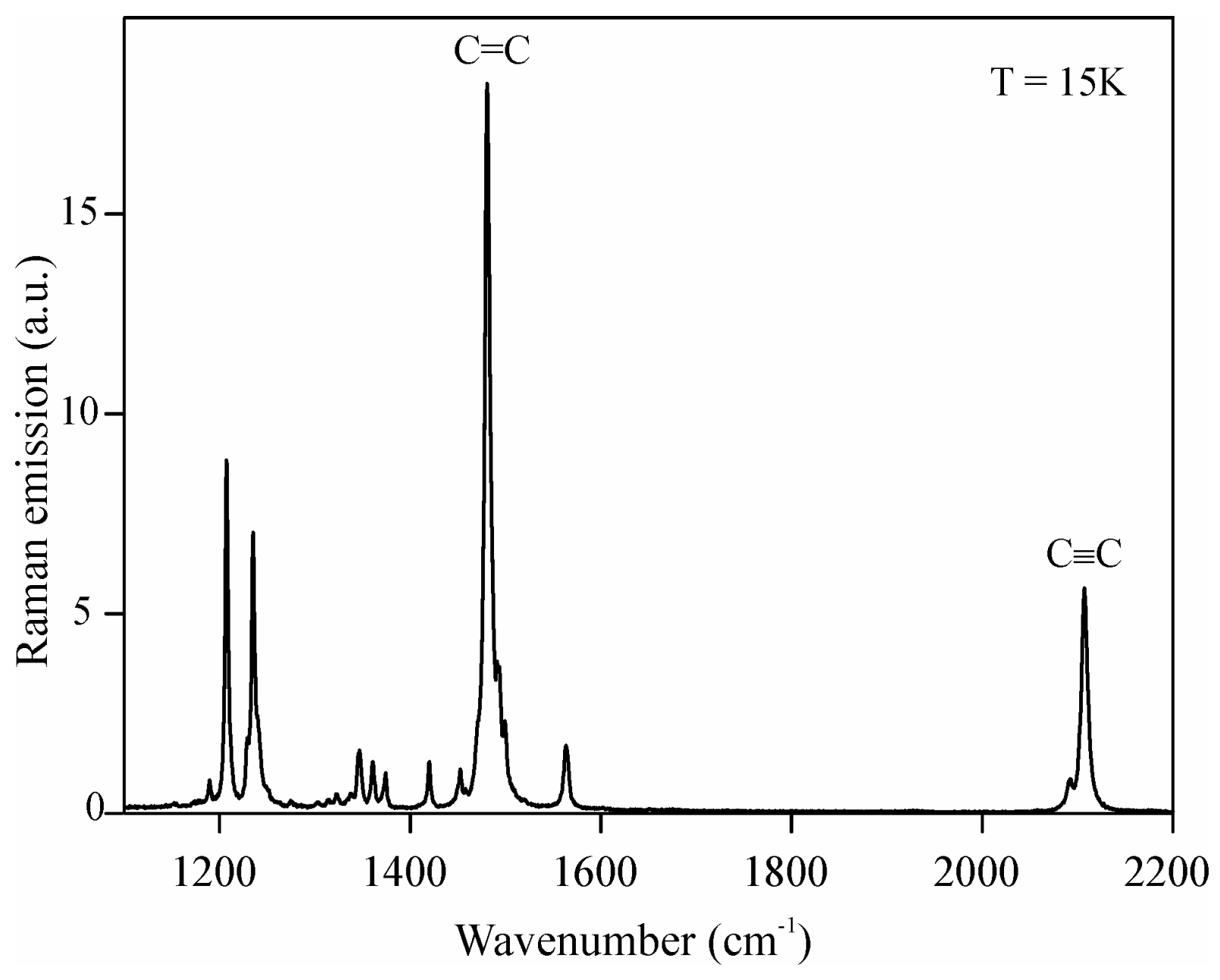

Figure 3 


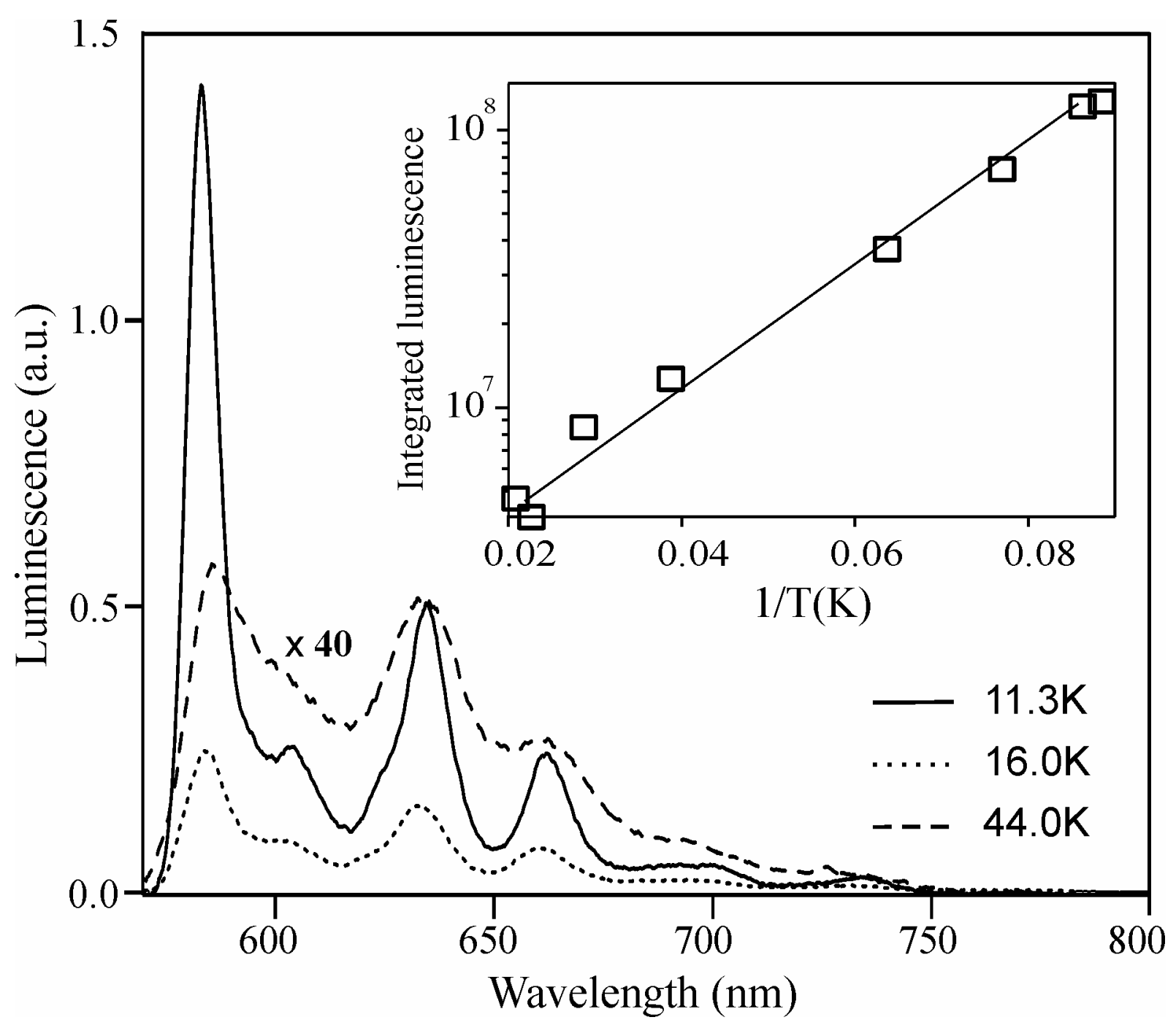

Figure 4 


\section{Table captions}

Table 1: Transition energies of the exciton and its satellites, taken from spectra of $\operatorname{Im}(\varepsilon)$ derived from the electroreflectance at low temperature. $\Delta \mathrm{E}$ refers to the energy difference to the exciton and yields the vibrationnal energies in good agreement with Raman modes measured at room temperature [9] and low temperature (this work).

\begin{tabular}{|c|c|c|c|c|}
\hline Assignment & $\begin{array}{c}\mathbf{E} \\
(\mathbf{e V})\end{array}$ & $\begin{array}{c}\Delta \mathbf{E} \\
(\mathbf{m e V})\end{array}$ & $\begin{array}{c}\text { Raman } \\
(\mathbf{2 9 8} \mathbf{~ K})\end{array}$ & $\begin{array}{c}\text { Raman } \\
(\mathbf{1 5} \mathbf{~ K})\end{array}$ \\
\hline Exciton E & $\mathbf{2 . 1 7 1}$ & - & - & - \\
\hline a & 2.175 & $5 .(0)$ & 4.7 & 5.0 \\
\hline b & - & - & 6.7 & 6.0 \\
\hline c & - & - & 19.2 & 19.1 \\
\hline d & 2.226 & $54 .(7)$ & 54.8 & 55.2 \\
\hline S & 2.260 & $89 .(2)$ & 86.9 & $87.5,87.0,86.2$ \\
\hline & 2.276 & $105 .(5)$ & 106.1 & 106.3 \\
\hline D $^{*}$ & 2.327 & $156 .(4)$ & $153.2,149.9$ & $153.3,149.8$ \\
\hline D & 2.356 & $185 .(2)$ & 184.1 & 183.8 \\
\hline T & 2.439 & $267 .(7)$ & 261.7 & 261.5 \\
\hline Bandgap & $\mathbf{2 . 7 2 7}$ & $\mathbf{5 5 6}$ & & \\
\hline
\end{tabular}

Table 1 